\title{
EL USO DE LA APLICACIÓN WEB EFFITCIENCY MEJORA LA ADHERENCIA AL TRATAMIENTO EN PACIENTES CON ALTERACIONES NEUROLÓGICAS. ESTUDIO PILOTO
}

\section{EFFITCIENCY WEB APPLICATION USE IMPROVES PHYSIOTHERAPY TREATMENT ADHERENCE IN PATIENTS WITH NEUROLOGICAL DISORDERS. PILOT STUDY}

Esparcia Jiménez, Ángel ${ }^{1}$ Inza Plà, Francesc ${ }^{2}$ Cortés-Amador, Sara ${ }^{3}$ Sempere-Rubio, Núria ${ }^{4}$

1. Licenciado en Ciencias de la actividad física y el deporte y Graduado en Fisioterapia por la Universidad de Valencia, Fisioterapeuta en el Instituto Valenciano de Recuperación Deportiva. E- Mail: angel.esparcia@gmail.com

2. Ingeniero Técnico Industrial esp. Electrónica Industrial por la Universidad Politécnica de Valencia, desarrollador web freelance y desarrollador front-end en Tuvalum Sports. Valencia. España. E-mail: francesc.inza@gmail.com

3. Dra. en Fisioterapia, Profesora Asociada, departamento de Fisioterapia, Universidad de Valencia y Co- Fundadora de Fundación ACAVALL. E-Mail: sara.cortes@uv.es

4. Profesora Asociada, departamento de Fisioterapia, Universidad de Valencia y fisioterapeuta en la Clínica Fisioterapia Marítim, Lirios Dueñas. E-Mail: nuria.sempere@uv.es

Esparcia Jiménez, Á., Inza Pla, F., Cortés-Amador, S. y Sempere-Rubio, N. (2016). El uso de la aplicación web effitciency mejora la adherencia al tratamiento en pacientes con alteraciones neurológicas. Estudio piloto. 3C TIC: Cuadernos de desarrollo aplicados a las TIC, 5(3), 1-7. DOI: <http://dx.doi.org/10.17993/3ctic.2016.53.1-7/>. 


\section{RESUMEN}

En la actualidad, el porcentaje de incumplimiento de las pautas de ejercicio físico en pacientes con problemas neurológicos supera el 50\%, teniendo esto un impacto negativo en la calidad de vida de los usuarios/as. La bibliografía disponible pone de manifiesto que las app pueden ser herramientas que fomenten la implicación de la persona en su proceso de rehabilitación (Østerås et al. 2014; Peña, Juan y Moreno, 2010). Siguiendo esta línea de investigación, se ha diseñado una aplicación web destinada a la gestión de rutinas de ejercicio físico de un centro de fisioterapia y además, se ha comparado el soporte para la gestión de las mismas: formato papel o formato electrónico favorece la adherencia al tratamiento.

\section{ABSTRACT}

Nowadays non-adherence in physiotherapy treatment in patients with neurological disorders stands at $50 \%$. Data suggests that web based applications could be useful to promote the patients involvement in their rehabilitation process ( $\varnothing$ sterås et al. 2014; Peña, Juan y Moreno, 2010). Following this research Effitciency has been designed, a web application that manages patients workout routines in a physiotherapy center. Additionally has been determinated which routine treatment presentation format, paper or App, improves treatment adherence.

\section{PALABRAS CLAVE}

Adherencia, Fisioterapia, rutina, App, papel.

\section{KEYWORDS}

Adherence, Physiotherapy, exercise-programme, App, paper. 


\section{INTRODUCCIÓN}

La Organización Mundial de la Salud (OMS) define la salud en línea electrónica (e-health), como el uso de las tecnologías de información y comunicación (TICS) en la salud, ocupándose de mejorar el flujo de información a través de medios electrónicos, para apoyar la prestación de servicios de salud y la gestión de sistemas sanitarios (World Health Organization, 2011). Ante ello, surge la necesidad de desarrollar confianza en las nuevas tecnologías, tanto entre los profesionales de salud como entre los pacientes y ciudadanos, dado que de esto dependerá el éxito en la implementación de las TICs (Galán-Rodas y Zamora, 2014). En el ámbito de las TICs, una aplicación móvil o app es un software o programa informático que está diseñado para funcionar en teléfonos inteligentes (smartphones), tablets y otros dispositivos móviles. La bibliografía científica muestra que las TICs son un medio también para la mejora de algunos aspectos en salud, como permitir la mejor gestión de las organizaciones, posibilitar un mejor aprovechamiento de los recursos, y brindar un servicio de mayor calidad a los usuarios (Montero, García y Ríos 2004; Norman et al., 2004; Oviedo y Fernández 2010).

"En fisioterapia es común prescribir actividades y ejercicios por periodos prolongados de tiempo, en ocasiones se dan las instrucciones de forma rápida y sin revisiones de mantenimiento que confirmen el ajuste al tratamiento del propio paciente", llegándose a no comprobar ni la comprensión ni la adecuada ejecución del ejercicio propuesto. Existen datos de estudios epidemiológicos de éste y otros países que indican que los pacientes incumplen las prescripciones alrededor del $50 \%$ de las veces en tratamientos de fisioterapia.

La bibliografía justifica la prescripción de ejercicio físico como una prioridad en la prevención de las manifestaciones de enfermedades crónicas degenerativas no transmisible. Una rutina de ejercicio físico adecuada mejora el pronóstico y la calidad de vida en fatiga por inactividad física, obesidad, diabetes, hipertensión, debilidad o alteraciones músculo esqueléticas y cardiovasculares (Zurita et al., 2009; Jiménez et al., 2014). No obstante, el trabajo de los profesionales de la salud pierde significado en el momento en el que no se aplica con la eficacia deseada en un paciente, es decir, una rutina de ejercicios realizada por fisioterapeutas, adaptada a las características del paciente y en busca de unos objetivos concretos se encuentra sesgada en la medida en que el paciente destinatario de la misma no la realiza de forma adecuada, ya sea por falta de comprensión o de motivación. Como sostienen Montero et al., (2004) "La mayoría de los pacientes que llegan a una consulta de Fisioterapia no son técnicos en la materia. Los profesionales de la salud tienden a explicar y dar instrucciones demasiado técnicas, esto hace que el paciente no entienda ni lo que pasa ni lo que debe hacer para mejorar su situación".

La OMS sostiene que la implicación activa de un paciente va a depender de cinco aspectos fundamentales: aspectos socioeconómicos, aspectos del sistema de asistencia sanitaria, aspectos relacionados con la enfermedad, y aspectos relacionados con el paciente. Si bien es cierto que desde la fisioterapia no se puede influir en todos estos factores, es importante incidir en aquellos en los que sí existe esta posibilidad. Los usuarios necesitan, además de una asistencia individualizada, componentes motivacionales que mantengan el hábito de seguimiento de una rutina de ejercicios, sin olvidar que nuestros pacientes pueden presentar 
hándicaps como bajos niveles de actividad física o dificultad de compresión de las explicaciones dadas por el fisioterapeuta, lo que condiciona negativamente la adherencia a este tipo de tratamiento. Niveles altos de cumplimiento y adherencia a los tratamientos fisioterapéuticos aumentan la efectividad de los mismos y se sabe que la comprensión, la motivación y la implicación activa de los pacientes son determinantes en la misma (Montero et al., 2004; Reed et al., 2015; World Health Organization, 2011).

El diseño de una aplicación web teniendo en cuenta todos estos factores puede ser una herramienta que facilite a la comprensión, mejore la implicación y motivación de los pacientes en su proceso de rehabilitación y, además, sea un instrumento que facilite la comunicación usuario-profesional. La comunicación y la relación terapéutica paciente terapeuta condicionan la adherencia al tratamiento. Es necesario un proceso interactivo de comunicación para la correcta relación terapéutica ( $\varnothing$ sterås et al. 2014; Peña, Juan y Moreno, 2010).

El objetivo general del presente trabajo es diseñar una aplicación web destinada a la gestión de rutinas de ejercicio físico de un centro de fisioterapia para poder comparar que soporte de gestión de rutinas: el formato papel o formato electrónico favorece la adherencia al tratamiento.

\section{METODOLOGÍA}

Se realizó un estudio de casos en el que participaron 8 usuarios del centro Tetrasport Wellness. Se seleccionaron a aquellos usuarios que cumplían con los siguientes criterios de inclusión:

- Tener prescrita una rutina de ejercicio físico en el centro Tetrasport Wellness.

- Estar en un proceso de rehabilitación funcional a largo plazo.

- Disponibilidad y voluntad personal.

- Poseer un Smartphone con versión de Android posterior a 4.0 y de IOS posterior a la 7.0.

- Ser capaz de contestar las 30 preguntas del cuestionario.

- Capacidad de concentración, atención, entendimiento y comunicación verbal y escrita.

- Finalmente, aceptar su participación en el estudio.

En total se incluyeron 8 usuarios, con una media de edad 33,8 años, siendo el $62.5 \%$ menores de 25 años y el $37.5 \%$ mayores de 50 años. La media de edad de los usuarios de la muestra es de 33.8 años, siendo el $62.5 \%$ menores de 25 años y EL $37.5 \%$ mayores de 50 años.

Para la medición de las variables de estudio: cantidad y comprensión de información, motivación y satisfacción, se elaboró un cuestionario ad hoc de 30 ítems, con 5 opciones de respuesta siendo 1 totalmente en desacuerdo y 5 totalmente de acuerdo. 
Los usuarios, durante tres semanas, realizaron sus rutinas de ejercicio teniendo como soporte de apoyo una hoja de papel. En ella podían visualizar un dibujo que representaba el ejercicio que debía llevar a cabo. Finalizado este periodo, el usuario cumplimentaba el cuestionario ad hoc. A continuación, a los mismos usuarios que habían usado el formato papel, se les enseñó el manejo de la aplicación. Una vez aprendido, el participante, realizaba su entrenamiento teniendo como apoyo la app Effitciency. Esta app permitía al usuario ver en la pantalla de su móvil un video en bucle de la ejecución del ejercicio, el material necesario para hacerlo, parámetros de cuantificación de la carga, así como un contador de tiempo de descanso sincronizado con el contado de series. Después de 3 semanas de utilización de la app, como apoyo para la realización de la rutina de ejercicio, el participante cumplimentaba el cuestionario.

Se utilizó el paquete estadístico SPSS versión 21.0 para el análisis los datos. De cada variable se realizó, en primer lugar, un análisis descriptivo de las medidas de tendencia central y en segundo lugar, un análisis inferencial empleando Pruebas $\mathrm{T}$ para muestras relacionadas, siendo el valor de significación $p<0.05$.

Todos los participantes del estudio cumplimentaron el consentimiento informado, en el que se les informó del desarrollo del estudio así como de los riesgos del mismo.

\section{RESULTADOS Y DISCUSIÓN}

En la Tabla 1, se reflejan los estadísticos descriptivos y la significación de las variables objeto de estudio, y en ella se puede observar que el soporte electrónico Effitciency obtiene en todas las variables de estudio una media superior a las del papel como recurso de apoyo. Además, cuando se comparan las medias obtenidas por el formato papel y las alcanzadas con el formato electrónico, se observa que la diferencia es estadísticamente significativa, ocurriendo esta situación en cada una de las variables analizadas.

Tabla 1. Análisis de las variables de estudio.

\begin{tabular}{|c|c|c|c|c|c|c|c|c|c|}
\hline Estadísticos & \multicolumn{3}{|c|}{ Motivación } & \multicolumn{2}{c|}{ Satisfacción } & \multicolumn{2}{c|}{$\begin{array}{c}\text { Comprensión } \\
\text { Información }\end{array}$} & \multicolumn{2}{c|}{$\begin{array}{c}\text { Cantidad } \\
\text { Información }\end{array}$} \\
\hline & Papel & App & Papel & App & Papel & App & Papel & App \\
\hline Media & 3,48 & 4,41 & 3,07 & 4,75 & 2,87 & 4,62 & 2,6 & 4,35 \\
\hline Mediana & 3,75 & 4,83 & 3,1 & 4,8 & 2,82 & 4,61 & 2,3 & 4,4 \\
\hline Desv. Típica & 0,73 & 0,34 & 0,92 & 0,29 & 0,62 & 0,19 & 0,71 & 0,41 \\
\hline Mínimo & 2,17 & 4,17 & 1,8 & 4,2 & 2 & 4,43 & 2 & 3,8 \\
\hline Máximo & 4,5 & 5 & 4,4 & 5 & 3,86 & 5 & 3,8 & 5 \\
\hline Significación & 0,005 & 0,002 & 0,000 & & 0,000 \\
\hline
\end{tabular}

Fuente: elaboración propia.

El desarrollo de TICs en el ámbito de la fisioterapia se encuentra todavía en estadios iniciales. Según la OMS, es necesario desarrollar confianza en las nuevas tecnologías, tanto entre los profesionales de salud como entre los pacientes y ciudadanos. Es necesario por tanto, tener una actitud favorable por parte de los profesionales que permita la integración de estas 


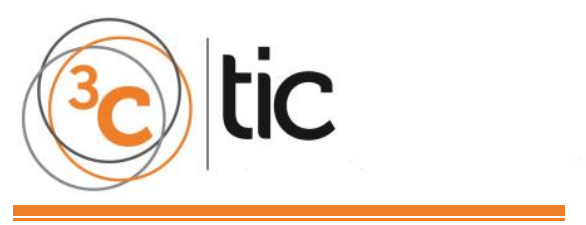

herramientas tanto en la investigación como en la experiencia clínica (World Health Organization, 2011).

Existen ejemplos de efectos beneficiosos en intervenciones basadas en Salud Electrónica, incluyendo entre otros efectos a la mejora de la adherencia a la actividad física. Según los resultados de este estudio, se puede observar también como un aumento en la cantidad y calidad de información proporcionada al paciente mejoras también en la satisfacción y motivación por la tarea, coincidiendo esta premisa con los resultados de este trabajo (Norman et al., 2004).

Según la OMS, existe una interrelación entre los diferentes factores que influyen en la adherencia a un tratamiento. El presente trabajo coincide con la premisa del trabajo de la OMS, donde concluyen que la compresión de la información es necesaria para lograr una adecuada motivación. Es complicado garantizar la implicación activa de un paciente en un proceso que desconoce, como afirman Montero et al. (2004), el conocimiento de las características de una enfermedad así como de la orientación del tratamiento permite mejorar la participación del paciente en el proceso.

No obstante, la comprensión del proceso por parte del paciente no garantiza su implicación, como afirman $\emptyset$ sterås et al. (2014) en su trabajo. Los usuarios necesitan factores motivantes que sustenten el hábito de cumplimiento de la prescripción de un tratamiento, quizá el uso de la aplicación diseñada puede ser un factor motivador en sí mismo. Existe evidencia de que la simple interacción con un Smartphone puede mejorar la motivación por una tarea y en consecuencia, la adherencia a una determinada intervención.

\section{CONCLUSIONES}

Los resultados del presente estudio reflejan que el uso de la app durante un periodo de tres semanas, a diferencia de las rutinas en formato papel, puede mejorar la adherencia a un tratamiento de ejercicio físico. Esta mejora se debe a que, cuando se analizan las puntuaciones de cada una de las variables de estudio: satisfacción, cantidad y comprensión de la información y motivación, en el formato informático (uso de la app), son significativamente superiores a las obtenidas por el formato en papel, siendo todas ellas elementos clave en la adherencia al tratamiento. No obstante, los resultados obtenidos no son extrapolables, puesto que el tamaño muestral y el tiempo de uso de ambos formatos son reducidos. Este trabajo, es un estudio piloto que refleja las posibilidades que las tecnologías de la información y comunicación podrían tener en el ámbito de la fisioterapia. Es necesario continuar esta línea de investigación para poder alcanzar resultados más concluyentes que permitan establecer el potencial de las TICs en este campo. 


\section{REFERENCIAS BIBLIOGRÁFICAS}

Galán-Rodas, E., y Zamora, A. (2014). La historia clínica electrónica como herramienta de gestión y mejora del proceso de atención de salud en Costa Rica. Acta Médica Costarricense, 56(1), 35-36.

Jiménez, C.E., Fernández, R., Zurita, F., Linares, D., y Farías, A. (2014). Programas de Educación en Salud y Entrenamiento de la Fuerza en adultos mayores con artrosis de cadera leve a moderada. Revista médica de Chile, 142(4), 436-442.

Montero, M., García, R.F., y Ríos, M.D. (2004). Un análisis de la adherencia al tratamiento en fisioterapia. Fisioterapia, 26(6), 333-339.

Oviedo, E., y Fernández, A. (2010). Tecnologías de la información y la comunicación en el sector salud: oportunidades y desafíos para reducir inequidades en América Latina y el Caribe. CEPAL.

Norman, G. J., Zabinski, M. F., Adams, M. A., Rosenberg, D. E., Yaroch, A. L., y Atienza, A. A. (2007). A review of eHealth interventions for physical activity and dietary behavior change. American journal of preventive medicine, 33(4), 336-345.

Peña, A. N., Juan, J. C., y Moreno, N. A. (2010). La interacción comunicativa en el cuidado de la salud. Rev Esp Com Sal, 1(2), 113-29.

Østerås, N., Hagen, K. B., Grotle, M., Sand-Svartrud, A. L., Mowinckel, P., Aas, E., \& Kjeken, I. (2014). Exercise programme with telephone follow-up for people with hand osteoarthritis-protocol for a randomised controlled trial. BMC musculoskeletal disorders, 15(1), 1.

Reed, J. L., Prince, S. A., Cole, C. A., Nerenberg, K. A., Hiremath, S., Tulloch, H. E., ... y Pipe, A. L. (2015). E-health physical activity interventions and moderate-to-vigorous intensity physical activity levels among working-age women: a systematic review protocol. Systematic reviews, 4(1), 1.

World Health Organization. (2011). Global Observatory for eHealth series. Retrieved March, 4, 2011.

Zurita Ortega, F., Fernández García, R., Cepero González, M., Zagalaz Sánchez, M. L., Valverde Cepeda, M., y Ramírez Domínguez, P. (2009). The relationship between pain and physical activity in older adults that begin a program of physical activity. 